\title{
Assessing Plastically Dissipated Energy as a Condition for Fatigue Crack Growth
}

D. Cojocaru

University of Delaware

Anette M. Karlsson

Cleveland State University, a.karlsson@csuohio.edu

Follow this and additional works at: https://engagedscholarship.csuohio.edu/enme_facpub

Part of the Mechanical Engineering Commons

How does access to this work benefit you? Let us know!

\section{Publisher's Statement}

NOTICE: this is the author's version of a work that was accepted for publication in International Journal of Fatigue. Changes resulting from the publishing process, such as peer review, editing, corrections, structural formatting, and other quality control mechanisms may not be reflected in this document. Changes may have been made to this work since it was submitted for publication. A definitive version was subsequently published in International Journal of Fatigue, 31, 7, (07-01-2009); 10.1016/j.ijfatigue.2008.12.009

\section{Original Citation}

Cojocaru, D., and Karlsson, A. M., 2009, "Assessing Plastically Dissipated Energy as a Condition for Fatigue Crack Growth," International Journal of Fatigue, 31(7) pp. 1154-1162.

This Article is brought to you for free and open access by the Mechanical Engineering Department at EngagedScholarship@CSU. It has been accepted for inclusion in Mechanical Engineering Faculty Publications by an authorized administrator of EngagedScholarship@CSU. For more information, please contact library.es@csuohio.edu. 


\title{
Assessing plastically dissipated energy as a condition for fatigue crack growth
}

\author{
D. Cojocaru, A.M. Karlsson * \\ Department of Mechanical Engineering, University of Delaware, 126 Spencer Laboratory. Newark, DE 19716, United States
}

\section{Introduction}

Fatigue of materials has imposed limitations on engineering structures since the beginning of the industrial revolution and even though tremendous research efforts have been aimed towards understanding and predicting fatigue, a reliable method to predict fatigue crack growth still does not exist [1-6]. Currently, there are several avenues proposed for modeling fatigue crack growth in order to obtain life prediction models, including using damage mechanics [7,8], stress intensity factors [9-12], and energy criteria [13-18] as prediction tools for crack propagation. Some of these approaches are enhanced with incorporating various forms of "cycle-jump" techniques, to accelerate the numerical simulations [1923].

In this work, we investigate the concept of using plastically dissipated energy as a criterion for crack propagation due to cyclic loading. The plastically dissipated energy can be directly linked to the accumulation of plastic strain, which in turn can for example be linked to accumulation of dislocation in metals. Rice [13] suggested fatigue crack growth using plastic dissipation as a criterion in 1967. Turner proposed using the rate of energy dissipation for defining ductile tearing resistance $[15,16]$. Using the finite element (FE) method, Klingbeil [17] proposed a technique (based on the work of Bodner et al. [14]) for predicting fatigue crack growth in terms of the per-cycle rate of plastic energy dissipated in the reversed plastic region (defined in Fig. 1). The approach is based on evaluating the conditions around the crack tip for a non-propagat-

\footnotetext{
* Corresponding author. Tel.: +1 302831 6437; fax: +1 3028313619.

E-mail address: karlsson@udel.edu (A.M. Karlsson).
}

ing crack. Klingbeil [17] initially used the technique for stationary cracks under mode I loading and the technique was later applied by Daily and Klingbeil $[24,25]$ for stationary cracks under mixed mode loading conditions.

Cojocaru and Karlsson [18] presented a numerical scheme (a modeling frame) where cyclic crack growth can be simulated using finite element analysis (FEA). The modeling frame allows the crack to propagate once a user-defined propagation criterion is satisfied. The total (i.e. accumulated) plastically dissipated energy in a predefined region in front of the crack-tip was suggested as one of many possible propagation criteria for FE simulation of fatigue crack growth. The proposed concept is based on that, from a continuum perspective, the fatigue crack advances by cyclic material degradation in the process zone (Fig. 1) associated with the cracktip (see, for example, Ref. [1]). If the material is ductile, then the degradation of the material in the process region is accompanied by significant plastic deformation (e.g., ductile metals and polymers). For metals, plastic deformation is associated with dislocation motion, which is associated with fatigue [13]. Therefore, the plastically dissipated energy in the crack-tip vicinity may be a suitable measure for evaluating crack propagation. In this computational scheme, the per-cycle crack propagation rate is not prescribed, but automatically obtained by probing the plastically dissipated energy in a user-defined domain ahead the current position of the crack-tip [18]. The FE simulations are conducted cycleby-cycle, i.e. the entire cyclic loading path is simulated. Even though cycle-by-cycle simulations may be computationally prohibitive for a complete simulation of high-cycle fatigue, explicitly studying the evolution of the crack due to cyclic loading is important in many cases, including obtaining crack propagation rates for 


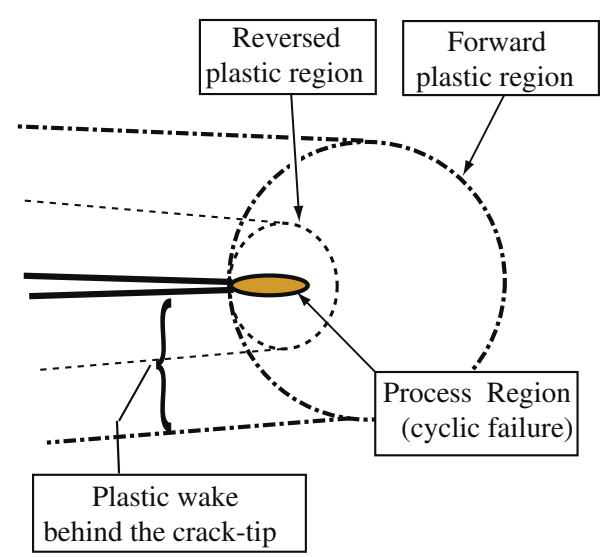

Fig. 1. Plastic regions developing during cyclic loading around a crack-tip and ensuing crack propagation (assuming macroscopic length scales). The forward plastic region forms during loading and the reverse plastic region develops when the load is reversed. The plastic wake develops when the crack propagates through the plastic region.

high-cycle fatigue and for cases where the growth of the crack influences the overall response (e.g., debonding of coatings). The proposed approach was shown to be able to capture both crack acceleration and crack retardation [18].

We will here qualitatively investigate if well established experimentally observed trends under selected forms of fatigue loading - including various load ratios and overloads - can be captured by a propagation criterion founded on the (accumulated) plastically dissipated energy. In this work, we focus on assessing this qualitatively via the numerical simulations. A quantitative evaluation can only be done by comparing with carefully developed experimental investigations. This will be done in a later study. The parameters introduced in Section 2 of this study (the "integrated domain" and "critically dissipated energy") are material parameters and thus will depend on the material investigated. The numerical simulations are based on the computational scheme developed previously [18]. The scheme uses a modeling frame for conducting FE simulations, where the crack propagation is simulated via a node-release technique. The main characteristics of this modeling frame are outlined in the next section.

\section{A numerical framework for simulating cyclic crack growth}

\subsection{General concepts}

The modeling frame used in this work was presented in Ref. [18] and the main concepts will be summarized here for clarity. The unique feature of this approach is that the crack extension per load cycle $(d a / d N)$ is not prescribed but is the output from the simulations.

The crack propagation is achieved by releasing (previously constrained) nodes along a predefined propagation path. Node-release techniques have frequently been used for FE simulations of fatigue crack growth, e.g. Ref. [26-29]. In addition to its conceptual simplicity for simulating crack propagation along predicted paths under cyclic loading, the node-release technique has the major advantage of avoiding time consuming re-meshing procedures. This is critical for simulating a large number of load cycles. Furthermore, the node-release approach allows using contact formulations for the crack surfaces.

The developed modeling frame uses ABAQUS Scripting Interface [30], where a FE model is generated automatically. The fundamental modeling idea is that any two-dimensional (2D) structure can be decomposed in two sets of (data) entities: (i) a set of continua and (ii) a set of continuum interfaces. A continuum represents a sub-domain having its own constitutive response, typically coded in a separate constitutive subroutine. There are two types of continuum interfaces describing (i) the interactions between two adjacent continua, or (ii) between a continuum and the exterior.

Central to simulating cyclic crack growth is the representation of the interface between two continua. This type of interface is represented as an arbitrary sequence of (i) failed (i.e. separated) segments (modeling cracks) and (ii) intact segments (modeling potential crack extension paths). The end vertices of the intact segments are potential crack-tips and can be assigned propagation criteria based on any quantity available in the FE analysis. An iterative evaluation procedure is called at the end of user prescribed intervals (measured in number of load cycles) to assess the propagation criteria and to advance the crack-tips when a propagation criterion is fulfilled. This modeling frame is a convenient research tool for modeling cyclic crack growth in a variety of cases. Applications of cyclic crack propagation in fracture mechanics specimens, multi-layered systems and structures with geometrically modeled micro-features are illustrated in Ref. [31].

\subsection{Crack propagation}

The crack propagation criterion investigated in this work is based on using the total (i.e. accumulated) plastically dissipated energy in a predefined region in the vicinity of the crack-tip. Evaluating the plastically dissipated energy only in the vicinity of the crack-tip (as opposed to, for example, evaluating the plastic work for the entire structure) is motivated by the concept of the "process region" (Fig. 1). The proposed approach allows the crack propagation to be assessed independently (for each crack-tip) in the case of multiple cracks. This was illustrated in Ref. [18] for a bi-layer structure containing two cracks.

The accumulated plastically dissipated energy is determined by evaluating this quantity in a discrete domain (the integrated domain, D) in front of the crack-tip. Here, the discrete domain is defined as a set of elements, $E_{D}$, approximating a theoretical semi-disk domain of radius $r$, centered at the current crack-tip, Fig. 2. The plastically dissipated energy in the integrated domain is defined by $W^{p}(\mathbf{D})=\sum_{e} \in E_{\mathbf{D}} w_{e}^{p}$, where $w_{e}^{p}$ represents the plastically dissipated energy within (one element, $e$, (computed by Gaussian

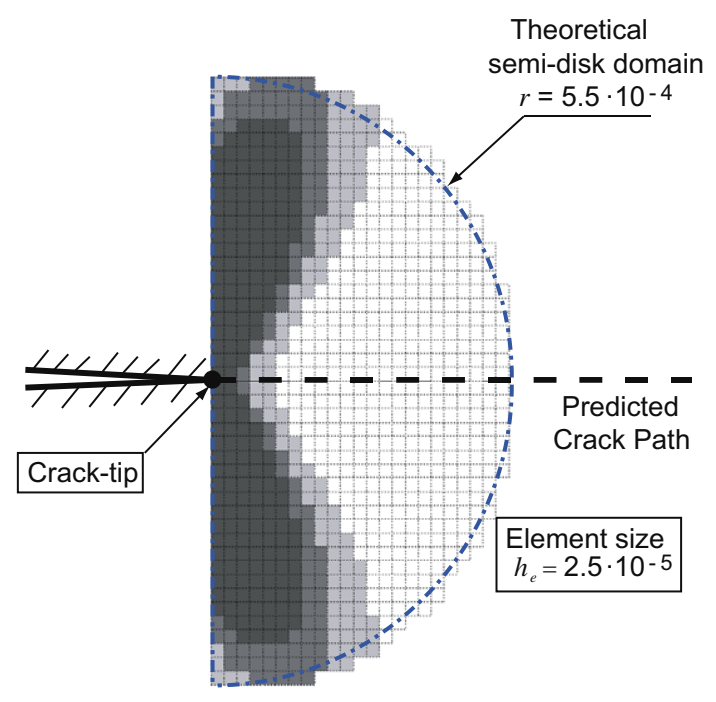

Fig. 2. The integrated (semi-disk) domain, $\mathbf{D}$, used by the propagation criterion, and the reversed plastic region after the first cycle (gray elements). Darker shades of gray indicate higher plastically dissipated energy in the reversed plastic region. 
quadrature using the density of plastically dissipated energy). The density of the plastically dissipated energy is calculated by the constitutive subroutine at each integration point. The discrete propagation rate per cycle after the $N$ th load cycle, $\left.\Delta a\right|_{N}$, is established by an iterative computation of the plastically dissipated energy over the integrated domain (here: the semi-disk domain). That is, the propagation rate is a result from the simulations and it is not prescribed. The rate $\left.\Delta a\right|_{N}$ is a discrete approximation of the continuous propagation rate $d a / d N$. The integrated domain is translated with one element length along the propagation path and the procedure is repeated until the value of the plastically dissipated energy in the current integrated domain, $W^{p}(\mathbf{D})$, is less than a user-specified critical value, $W_{c r}^{p}$. The iterative procedure for establishing the discrete propagation rate, $\left.\Delta a\right|_{N}$, is described in Ref. $[18,31]$. In this work, we assume that the critically dissipated energy, $W_{c r}^{p}$, is constant, since there is (to the knowledge of the authors) no experimental data available to quantify such parameter. Moreover, the size of the integrated domain (i.e. radius $r$ ) is based on the size of the reversed plastic region. Investigations on the shape, size and position of the integrated domain with respect to the crack-tip, and the appropriate value for the critically dissipated energy will be address in a future study where numerical studies are combined with extensive and careful experimental work.

\section{Model description}

In this study, we simulate fatigue crack growth in a plane strain specimen with a center crack. All dimensions are normalized, since the aim of the study is to qualitatively asses the usefulness of the proposed method. Thus the half width of the specimen is assumed $B / 2=1$ and the initial half length is $a=0.20$, Fig. 3A. Only the right side of the specimen is modeled since symmetry along the central vertical axis is assumed, with appropriate boundary conditions imposed. The boundary conditions are shown in Fig. 3A. Linear elastic, perfectly plastic constitutive response is used for all simulations, with the normalized yield strength $\sigma_{\mathrm{ys}}=1$, the normalized elastic modulus $E=350$, and Poisson's ratio $v=0.3$. Bi-quadratic plane strain elements with reduced integration were used for the entire model. The $J_{2}$ computational plasticity theory $[32,33]$ is used to predict the inelastic material response including the density of the plastically dissipated energy. The specimen is subjected to cyclic stress applied to the top and bottom edges (Fig. 3). During each cycle, the stress is varied linearly from an initial minimum value, $\bar{\sigma}_{\min }$, to a maximum value, $\bar{\sigma}_{\max }$, and back to the initial value, $\bar{\sigma}_{\min }$. Here, $\bar{\sigma}_{\max }=1 / 6$ unless otherwise specified. An important parameter used to characterize the cyclic load is the so-called load ratio, defined as $R=\bar{\sigma}_{\min } / \bar{\sigma}_{\max }$ [6]. In the benchmark cases considered in the following, we use $R=0\left(\bar{\sigma}_{\min }=0\right)$, thus the load cycle has only a tensile character, and $R=-1$, where the load is fully reversed from tensile to compressive during one load cycle $\left(\bar{\sigma}_{\min }=-1 / 6\right)$.

The crack propagation is simulated using the procedure summarized in Section 2 [18]. A refined structured mesh is used in a rectangular region $\left(1.35 \times 10^{-2}\right.$ by $\left.1.3 \times 10^{-3}\right)$ along the simulated crack path, Fig. $3 \mathrm{~A}$. In the refined mesh, all elements have the same size. In the current simulations, the element length in this structured mesh is $h_{e}=2.5 \times 10^{-5}$. This choice of element length concurs with the suggestion of Solanki et al. [28], who recommended to include at least 3-4 elements along the reversed plastic region (which will be verified later in the discussion). As shown in Ref. [18], the discrete propagation rate incurred after the $N$ th load cycle, assumes values of the form $\left.\Delta a\right|_{N}=k h_{e}, k=0,1,2 \ldots$, where $k$ is an integer resulting from the iterative updating procedure. The propagation rate, $\left.\Delta a\right|_{N}$, is the dis-
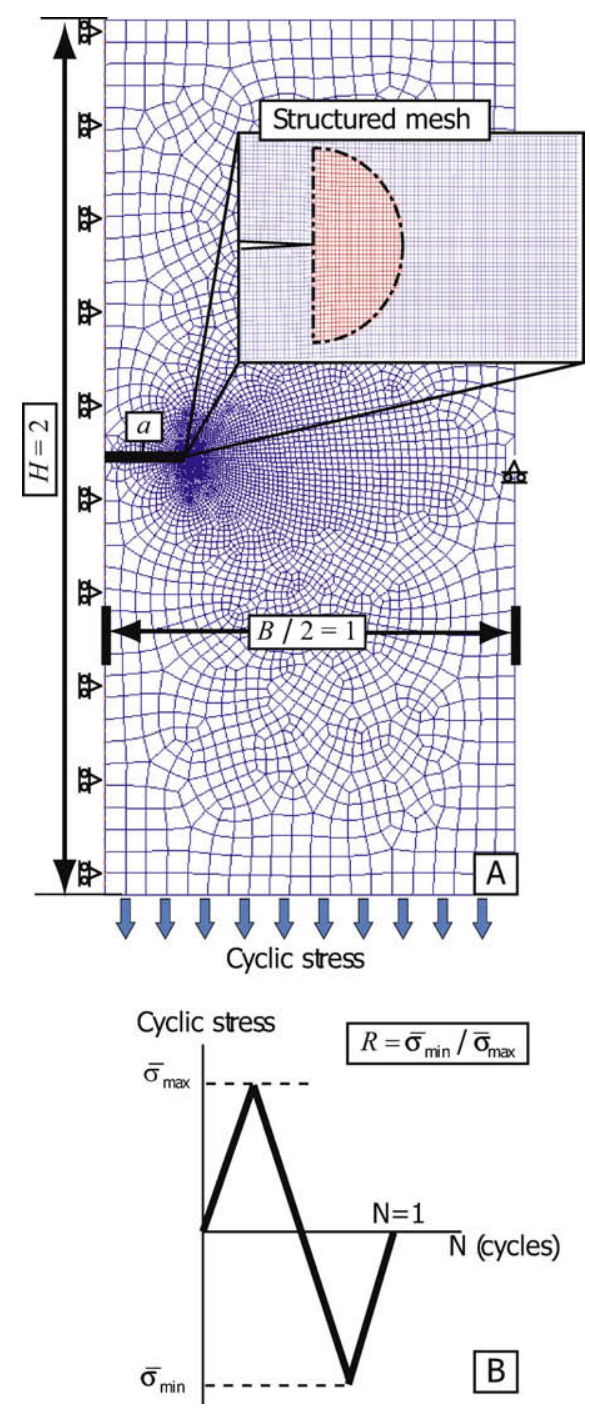

Fig. 3. (A) Finite element model of the investigated specimen where a refined structured mesh is used along the simulated crack path and (B) schematics of the load cycle.

crete equivalent of the fatigue crack growth rate $d a / d N$. The crack propagation rate is determined according to the following scheme (Section 2.2) [18]: A discrete semi-disk domain (the integrated domain), D, of radius $r$, (Fig. 2) is defined in front of the crack-tip. The plastically dissipated energy is evaluated in this domain after each cycle, $W^{\mathrm{p}}(\mathbf{D})$. Crack propagation ensues when a critical value, $W_{c r}^{p}$, is reached, $W^{p}(\mathbf{D}) \geq W_{c r}^{p}$. Since this is a new criterion, there is no procedure for selecting the radius, the shape and/or the position of the integrated domain with respect to the crack-tip. The goal of this paper is to assess the suitability of using the plastically dissipation energy as a propagation criterion from a qualitative perspective. Further numerical and experimental work is necessary to calibrate such a criterion. Since both $W_{p}^{\text {cr }}$ and $\mathbf{D}$ are material parameters, these will depend on the material investigated. The size and the shape of the integration domain considered here are obtained from preliminary analytical and numerical evaluations. In this initial study, we correlate the size of the semi-disk domain to the size of the reversed plastic region ${ }^{1}$ (Fig. 1) where significant plastic deformation occurs during both loading and unloading. The

\footnotetext{
${ }^{1}$ Integrated domains of more complex shapes can be developed using other models for the plastic region if desired.
} 
size and shape of the reversed plastic region can be estimated based on one of the various analytical models existing in the literature, or from numerical simulations.

For example, a simple analytical approximation of the size of the cyclic plastic region (in the direction of propagation) for a linear elastic, perfectly plastic material, subjected to mode I loading, can be obtained using the well-known relation for plane strain conditions $^{2}$ (see for example Ref. $[5,6]$ ):

$r_{c}=\frac{1}{3 \pi}\left({\frac{\Delta K_{I}}{2 \sigma_{y s}}}^{2}\right.$

where $r_{c}$ is the size of the reversed plastic region (measured from the crack tip), $\sigma_{\mathrm{ys}}$ is the material yield strength, and $\Delta K_{I}=K_{I}\left(\bar{\sigma}_{\max }\right)-K_{I}\left(\bar{\sigma}_{\min }\right) . K_{\mathrm{I}}(\sigma)$ is the stress intensity factor (SIF) for mode I of crack opening at the stress $\sigma$. For the specimen considered here (Fig. 3A), the mode I SIF can be computed using the formula due to Feddersen [34]:

$K_{I}(\sigma ; a, B)=\sigma \sqrt{\pi a}\left(\left(\sec \frac{\pi a}{B}\right)^{1 / 2}\right.$

In equation Eq. (2), $\&$ is the applied stress, $a$ is the half length of the crack and $B$ is the specimen width. For $\bar{\sigma}_{\min }=0, \bar{\sigma}_{\max }=\leftarrow$ $1 / 6, a=0.20$ and $B=2$ we obtain $\Delta K_{I}=0.1355$. Assuming $\sigma_{y s}=1$, the size of the reversed plastic region in front of the crack according to the equation Eq. (1) is $r_{c}=4.867 \times 10^{-4}$.

Alternatively, one can easily select the value of the radius based on a preliminary analysis with a single load cycle. For the load conditions listed above, we found that a semi-disk of radius $r=5.5 \times 10^{-4}$ covers most of the reversed plastic region in front of the crack tip ${ }^{3}$, generated during the described load cycle for the considered initial crack length (i.e. $a=0.2$ ). The value obtained from the numerical analysis is close to the analytical value, and we will use the value obtained from the numerical approach. The integrated domain is described as the set of elements that have at least one node within the theoretical semi-disk domain, Fig. 2 [18].

During cyclic loading, part of the crack surfaces (defined as the "failed segments" above) may be under contact. Thus, contact between the surfaces must be considered in the model. Contact occur not only for negative load ratios (i.e., $\bar{\sigma}_{\min }<0$ ), but can occur for cases where the load ratio vanishes or is positive (i.e., $\bar{\sigma}_{\min } \geq 0$ ). For these latter cases, contact is typically induced by what is commonly referred to as "plasticity induced crack closure" (PICC) and is associated with the plastic zone, the reverse plastic zone, and the plastic wake. Many authors have studied this phenomenon. It is generally agreed upon that PICC and the plastic zone directly affect the crack propagation rate; in particular it appears to slow the crack growth rate down, since PICC effectively reduces the stress intensity and may blunt the crack. This is automatically captured in our model (within the context of the formulation). For a review pertaining to modeling aspects on the subject, see Solanki et al. [35], and more recent work can be found for example in Refs. [36,37]. In our model, the normal, frictionless contact formulation in ABAQUS [38] was employed.

Cyclic crack growth is simulated for 100 cycles. ${ }^{4}$ Effects of (i) propagation criterion, (ii) load ratio, (iii) single overloads, and (iv) repeated overloads on the simulated fatigue crack growth, based on

\footnotetext{
${ }^{2}$ A similar relation but for plane stress conditions was initially credited to Rice [13].

3 The density of plastic dissipation (and so the equivalent plastic strain) has its absolute maximum within the first two elements immediately behind the crack-tip. Because these elements participate in "crack-tip blunting", the dissipated energy within these elements may exhibit mesh dependency due to excessive distortion. Therefore, we position the integrated domain so that only the elements in front of the crack-tip are included.

4 Simulating more cycles is computationally prohibitive on our single processor PC work station, due to the refined mesh used along the propagation path.
}

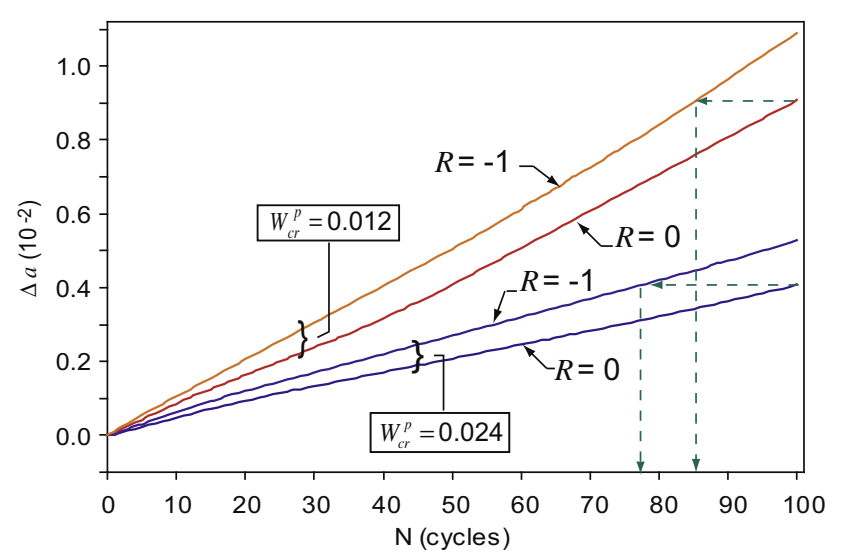

Fig. 4. The change in half crack length, $\Delta a$, as a function of the number of cycles, $N$, for two load ratios and two critical values of the plastically dissipated energy. The dashed lines indicate the reduction in the lifetime when $R=-1$ compared to $R=0$.

plastically dissipated energy evaluated in a semi-disk domain in front of the crack tip $\left(r=5.5 \times 10^{-4}\right.$ for all simulations $)$ are presented next.

\section{Numerical simulations: results and discussion}

\subsection{The effect of the propagation criterion}

First, we investigate the effect the propagation criterion has on the crack propagation, considering two magnitudes of the propagation criterion: $W_{c r}^{p}=0.024$ and $W_{c r}^{p}=0.012$. We note that due to the lack of suitable experimental data, these values were selected based on preliminary numerical investigations aiming to achieve a reasonable propagation rate within the context of the present purpose. Thus, we do not claim that these are physically realistic values, but are selected to demonstrate the concepts investigated here. In ongoing studies, realistic values are extracted. The load ratio $R=0$ with $\bar{\sigma}_{\min }=1 / 6$ is assumed. Ongoing experimental investigations will provide realistic values of both the propagation criterion in conjunctions with the size and shape of the integrated domain.

The resulting crack propagation is almost linear for both cases (Fig. 4). As expected, the crack propagates faster for the lower critical value of the plastic dissipation. The crack advances in increments of 1 or 2 elements per cycle when $W_{c r}^{p}=0.024$ and in increments of 3 or 4 elements per cycle when $W_{c r}^{p}=0.012$, Fig. 5 . The propagation rate, $\left.\Delta a\right|_{N}$, decreases after the initial 16 cycles when $W_{c r}^{p}=0.024$ and after 9 cycles for $W_{c r}^{p}=0.012^{5}$. We believe this decrease is caused by the formation of the plastic wake behind the crack-tip (associated with PICC as discussed above). The plastic wake forms as the crack propagates through the plastic region that has developed around the crack-tip during the loading-unloading sequence (Fig. 2). The plastic wake can effectively reduce the crack driving force for fatigue crack growth, see for example the review by Solanki et al. [35]. In contrast, one can observe an increase in propagation rate after 37 for the case $W_{c r}^{p}=0.012$ and after 77 cycles for $W_{c r}^{p}=0.024$. As the crack advances, the size of the plastic region, and energy dissipated per cycle in the vicinity of the crack-tip increase. This is captured by the iterative evaluation procedure of the propagation criterion and it results in larger propagation increments (i.e. crack acceleration).

\footnotetext{
${ }^{5}$ When assessing the evolution of the discrete propagation rate at a specific cycle number $N$, one should consider the discrete value at that cycle but also a sequence of several values occurred before, at and after $N$.
} 

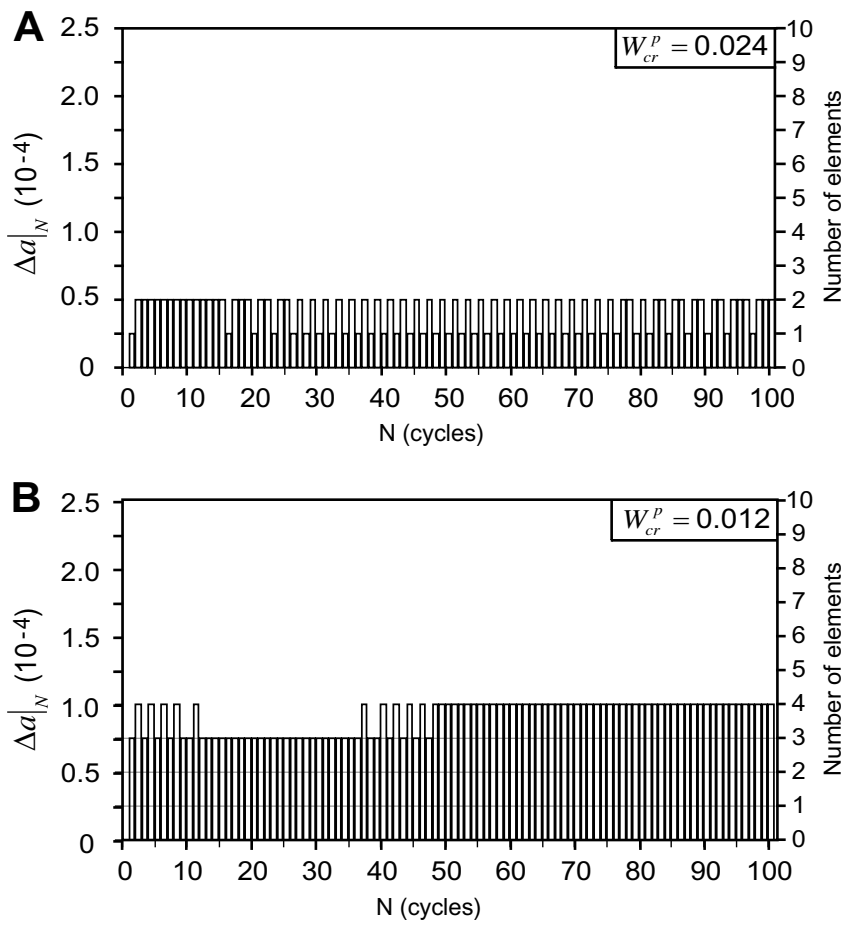

Fig. 5. Discrete propagation rate, $\left.\Delta a\right|_{N}$, with the corresponding number of elements (right ordinate), as a function of the number of cycles, $N$, for (A) $W_{c r}^{p}=0.024$, and (B) $W_{c r}^{p}=0.012$. (the element length is $h_{e}=2.5 \times 10^{-5}$ ).

Further insight to the crack propagation can be obtained by studying the normalized dissipated energy, Fig. 6, which is probed after the end of each cycle (during the first iteration of the iterative scheme [18]) in the discrete semi-disk domain, $\mathbf{D}$, ahead of the crack-tip. The normalized dissipated energy is defined by

$W_{c r}^{p}(\mathbf{D})=W_{c r}^{p}(\mathbf{D}) / W_{c r}^{p}$

Thus, the propagation criterion is fulfilled for any load cycle with $W_{n}^{p}(\mathbf{D}) \geq 1.0$, and the crack propagates at least one element. The oscillations in the plastically dissipated energy correspond to a crack propagating with an alternating number of elements for consecutive cycles. Thus, the crack propagates with $k$ elements after cycle $N$, with $k+1$ elements after cycle $N+1$ and again with $k$ elements after cycle $N+2$. In these transitory regimes, due to the discrete nature of propagation associated with the finite element, the integrated domain maybe advanced too far. Therefore, the plastically dissipated energy needs to accumulate over the next cycles until the criterion is fulfilled again. For example, in Fig. 5A for the cycles between 20 and 90 , the crack propagates in one cycle with one element length and in the following cycle with 2 elements. This is manifested with the oscillation in Fig. 6A. The smooth portions in the evolution of the dissipated energy are specific to the crack advancing at constant rate. This can be clearly seen by correlating the evolution of the discrete propagation rate (Fig. 5) to that of the dissipated energy for $R=0$ (Fig. 6).

\subsection{Effect of the load ratio}

Next, we investigate the effect of the load ratio on crack propagation. It is established from experimental work that load cycles with negative load ratio are associated with faster fatigue crack growth, e.g., [39]. We verify here that a propagation criterion based on plastically dissipated energy can capture this effect. We compare the two cases of critical values $W_{c r}^{p}=0.024$ and $W_{c r}^{p}=0.012$ for two load ratios: $R=0$ (i.e., $\left.\bar{\sigma}_{\min }=0\right)$ and $R=-1\left(\bar{\sigma}_{\min }=-1 / 6\right)$.
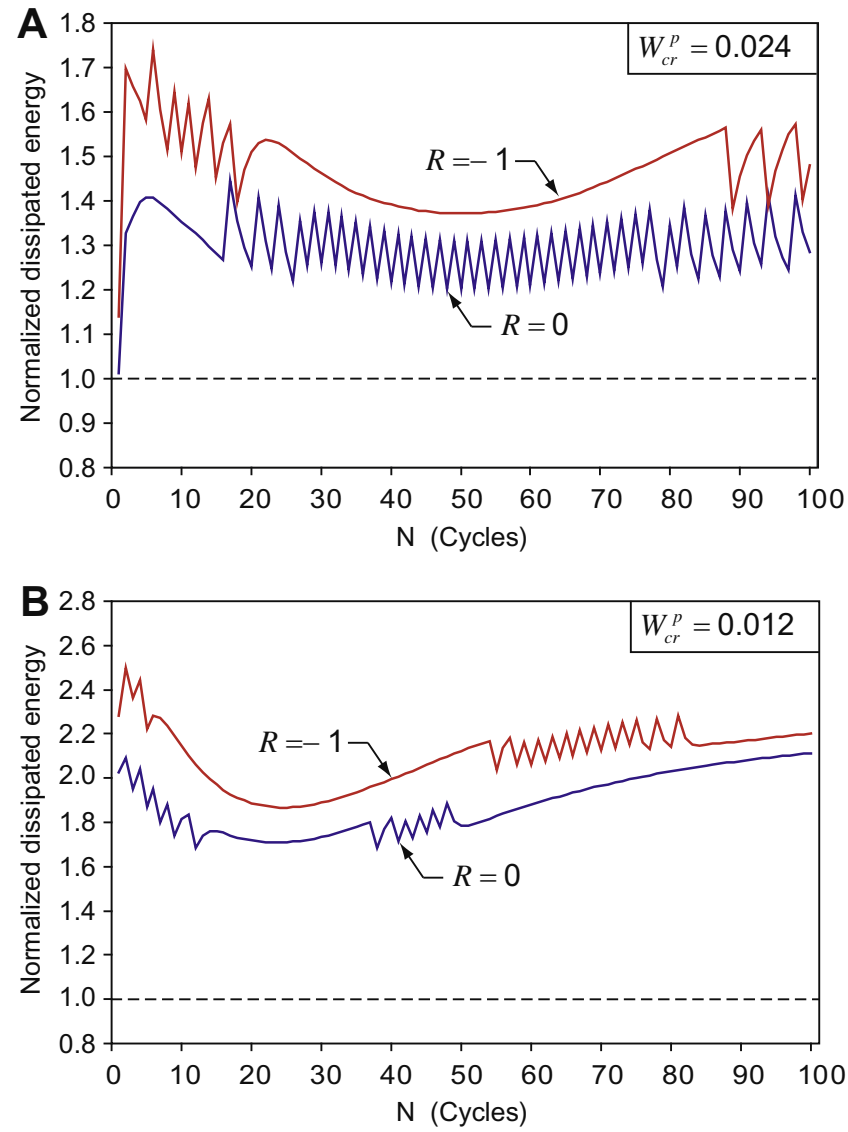

Fig. 6. Normalized dissipated energy in the semi-disk domain, $W_{n}^{p}(\mathbf{D})$, after each completed load cycle, as a function of the number of cycles, $N$, for $R=0$ and $R=-1$ when $(\mathrm{A}) W_{c r}^{p}=0.024$, and (B) $W_{c r}^{p}=0.012$

For all cases the maximum stress is the same, $\bar{\sigma}_{\min }=1 / 6$. and therefore the load for $R=-1$ is twice the load range than for $R=0$.

For negative load ratios, $R=-1$, the energy dissipated in the integrated domain is higher than for the cases with $R=0$ (Fig. 6). The increase in plastically dissipated energy for $R=-1$ leads to higher propagation rate, $\left.\Delta a\right|_{N}$, and consequently shorter crack growth life than for $R=0$ (Fig. 4). This agrees with experimental observations, which associate negative load ratios with higher fatigue crack growth rates and shorter lifetimes. For example, Stephens and co-workers [39] found that the fatigue crack growth life when $R=-1$ is about $88 \%$ of the crack growth life for $R=0$, for modified compact specimens made from 2024-T3 and 7075-T6 aluminum alloys. In our simulations, for $W_{c r}^{p}=0.012$, the crack length after 100 cycles for the case $R=0$ is achieved in about 85 cycles when $R=-1$. Similarly, for $W_{c r}^{p}=0.024$ the final crack length for the case $R=0$ is obtained in about 77 cycles when $R=-1$ (Fig. 4). Thus, our numerical results agree qualitatively very well with the experimental observations, clearly showing the same tendencies.

\subsection{Effect of a single overload}

In this section, we investigate how the criterion based on plastically dissipated energy captures the effect of a single tensile overload. The magnitude of the overload is characterized by the overload factor (or "overload ratio"),

$f_{0}=\bar{\sigma}_{\text {overload }} / \bar{\sigma}_{\text {max }}$.

It is well established experimentally that tensile overload cycles induce crack retardation, where the plastic deformation including crack tip blunting is commonly considered the major cause of 


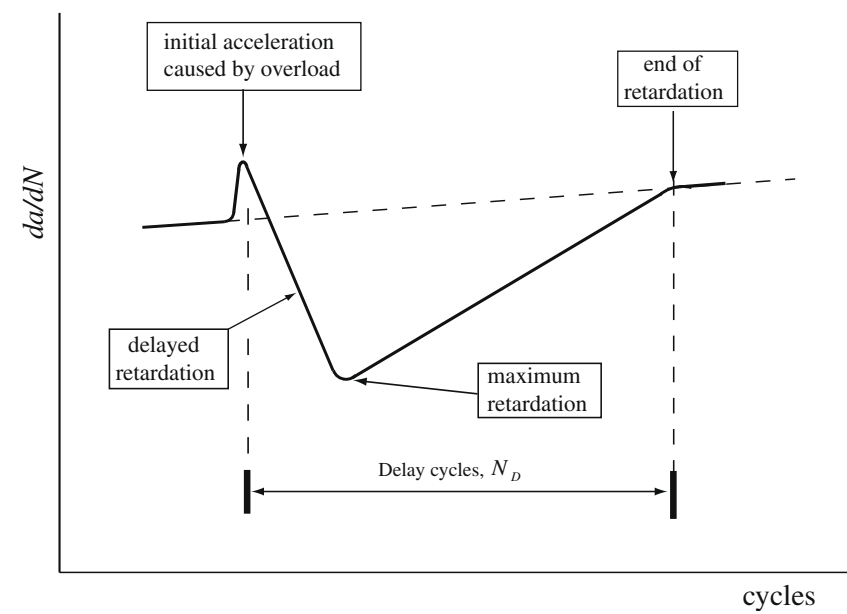

Fig. 7. Schematic representation of the crack propagation rate as a function of load cycles showing the effect of single tensile overloads on the crack growth rate, based on Refs. $[4,6]$.

the slow down [4,6,40-45]. Interestingly, the maximum crack retardation does not occur immediately after the overload, but after some additional cycling, $N_{D},[4,6,40-45]$. A schematic representation of the effect of a single tensile overload on the fatigue crack growth rate following those presented in the literature (e.g., Refs. [4,6]) is shown in Fig. 7. Verma and Pandey [44] found from experiments on center crack tension panels of 2024-T3 Aluminum-alloy, that the number of delay cycles due to overload, $N_{D}$, (Fig. 7) increases significantly with the increase of the overload factor, $f_{0}$. Vardar [42] performed overload tests on 7075-T6 Alclad sheets and found an exponential influence of the overload factor on the fatigue crack retardation.

We will here investigate if the proposed propagation criterion can capture these experimentally observed results. The simulations presented here are conducted for the case when the critical plastic dissipation is $W_{c r}^{p}=0.012$, the load ratio is $R=0$ with $\bar{\sigma}_{\max }=4 / 6$, and we will probe the results for single overloads of overload factors ranging from $f_{0}=1.0$ to 2.0. The single overload is applied during the 10th load cycle. As discussed in sub-section 4.1 , for this case $\left(W_{c r}^{p}=0.012\right.$ ), a sufficient plastic wake forms during the first 9 cycles leading to a "stabilized" discrete rate of propagation.

Crack profiles after 100 cycles are compared in Fig. 8, for $f_{0}=1.35$ and $f_{0}=1.75$ showing the integrated domain at the final crack-tip position. In this plot, the marks produced by the overloads can be clearly distinguished. Overload marks can also be ob-

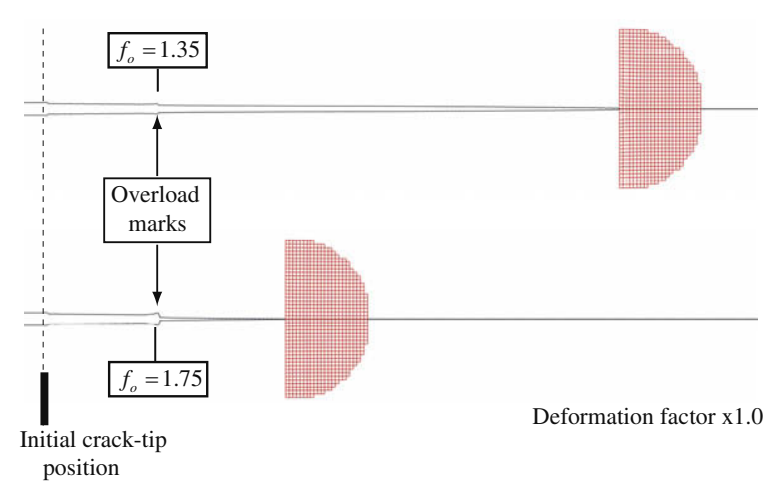

Fig. 8. Crack profiles after 100 cycles showing the marks created by a single overload applied during the 10th cycle. The discrete semi-disk domain is centered at the final crack-tip position. (The deformations are drawn to scale $\left(W_{c r}^{p}=0.012\right)$ served experimentally, see for example Ref. [41]. The overload mark may contribute to a discontinuous closure of the crack as the crack propagates [43].

The normalized dissipated energy in the discrete semi-disk domain, $W_{n}^{p}(\mathbf{D})$ (probed after each completed load cycle), is presented in Fig. 9A, for various overload factors. When the overload is applied, the plastically dissipated energy decreases significantly in the cycles that follow. The plastically dissipated energy decreases with increasing overload factor. Overload factors higher than 1.5 result in intermittent crack propagation, i.e. the crack does not propagate after each cycle. (When $W_{n}^{p}(\mathbf{D}) \ll 1.0$ the crack does not propagate and the plastically dissipated energy accumulates over several cycles before crack propagation occurs.) The numerical results also clearly indicate that the overloads cause crack retardation, Fig. 9B. Increasing the overload factor decreases the crack propagation rate which is in agreement with many experimental observations, see for example Ref. [44].

The effect of a single tensile overload can further be analyzed by studying the discrete crack propagation rate, $\left.\Delta a\right|_{N}$, (Fig. 10) and comparing to the absence of overloads (Fig. 5B). In the cycles following the overload, the crack growth rate first resumes to the value preceding the overload and then decreases. The maximum crack retardation does not occur immediately after the overload, but after some additional cycling, $N_{D}$. This delay in attaining maximum decrease in crack growth rate has been observed experimentally, as discussed above [4,6,40-45]. Thus, the simulations show that the features associated with crack retardation (Fig. 7) are captured during the simulation, including the delay, $N_{D}$, and the increase of $N_{D}$ with increasing overload factor. The delay in attaining maximum decrease in crack growth rate is best visible for $f_{0}=1.25$ (Fig. 10A). However, the maximum crack retardation
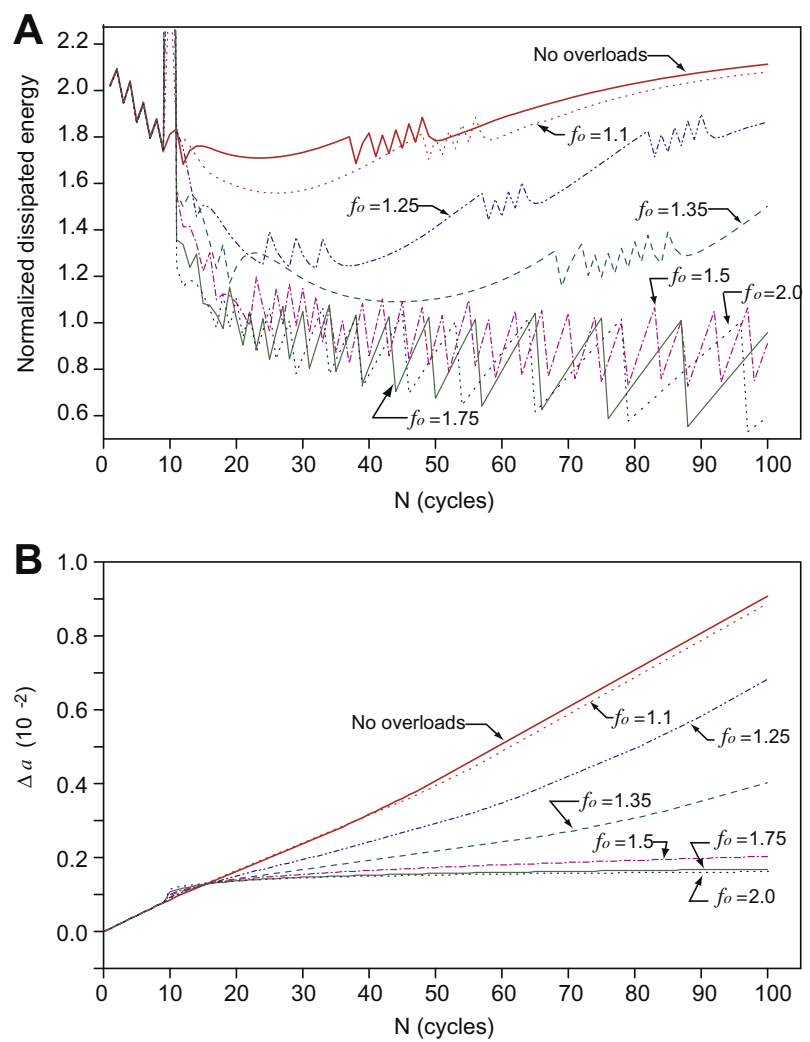

Fig. 9. Effect of a single tensile overload, $f_{0}$, of various magnitudes, on (A) normalized dissipated energy, $W_{n}^{p}(\mathbf{D})$, after each completed cycle; and (B) change in half crack length, as functions of the number of cycles, $N\left(W_{c r}^{p}=0.012\right)$. 

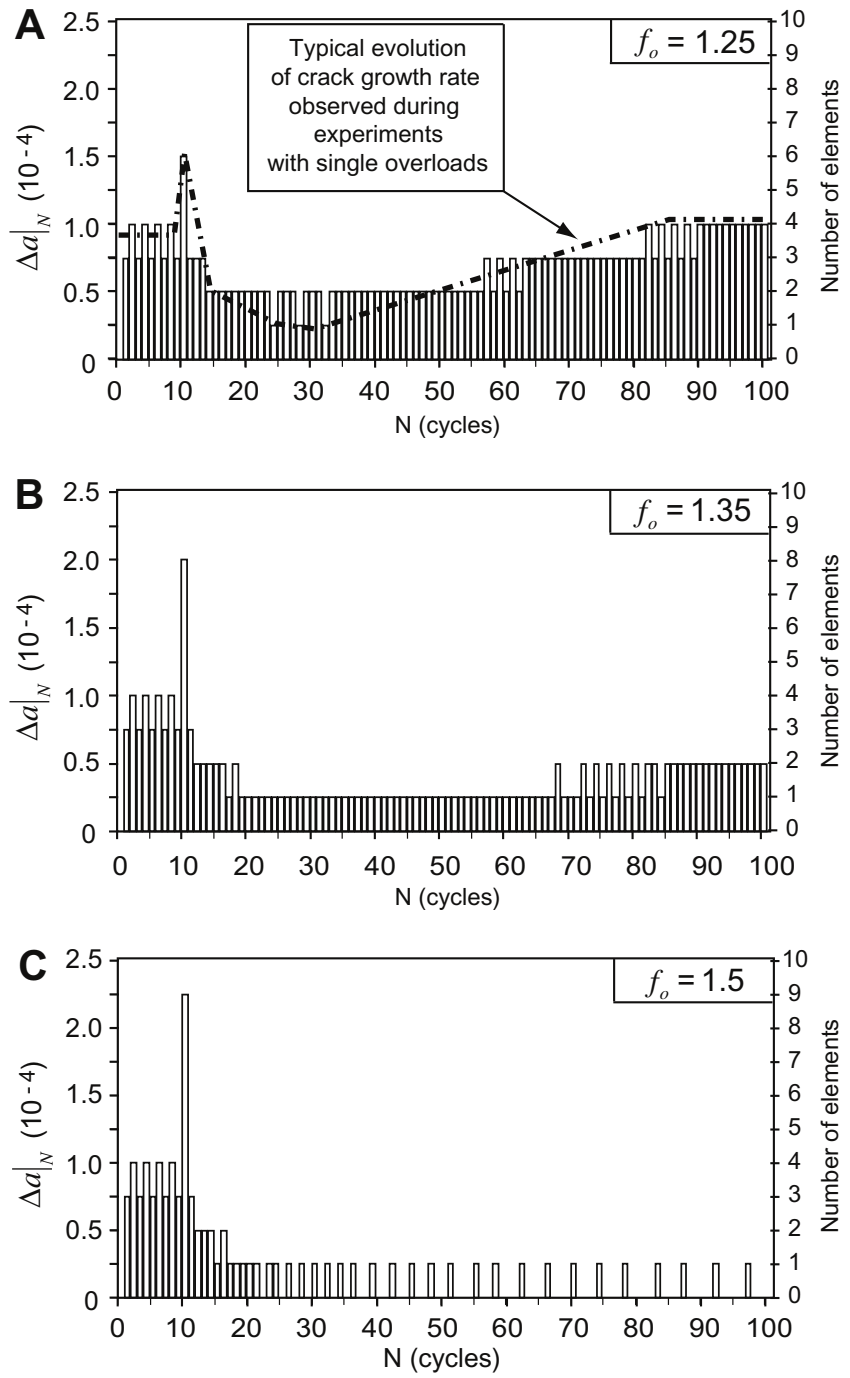

Fig. 10. Discrete propagation rate, $\left.\Delta a\right|_{N}$, as a function of the number of cycles, $N$, with the corresponding number of elements (right ordinate), when the overload factor is (A) $f_{0}=1.25$, (B) $f_{0}=1.35$ and (C) $f_{0}=1.5$. (The element length is $h_{0}=2.5 \times 40^{-5}$.) The dashed line in (A) indicate a typical evolution of the crack growth rate observed during experiments with single overloads $[4,6]\left(W_{c r}^{p}=0.012\right)$.

is not attained during the 100 simulated cycles when $f_{0} \geqslant 1.5$, indicating that the delay time increases with the overload factor.

Thus, the crack retardation behavior as captured by the proposed propagation criterion based on the plastically dissipated energy is in agreement with experimental observations, [4,6,40-45].

\subsection{Effect of repeated overloads}

Lastly, we investigate the effect of repeated overloads, where we first consider the response due to repeated overloads applied at different time intervals, $\Delta N$, and then the effect of critically dissipated energy associated with the propagation criterion.

When investigating the repeated overloads at different time intervals, a constant overload factor, $f_{0}=1.25$, is used. As for the previous cases, the critical plastic dissipation is $W_{c r}^{p}=0.012$, and the load ratio is $R=0$ with $\bar{\sigma}_{\max }=4 / 6$. The first overload is applied during the 10th cycle. Following the initial overload, we study the response ensuing when overloads of the same magnitude $\left(f_{0}=1.25\right)$ are repeated at intervals of 10,20 and 40 cycles. The normalized dissipated energy (obtained during the first iteration of the iterative procedure) is shown in Fig. 11A, whereas the evolu-
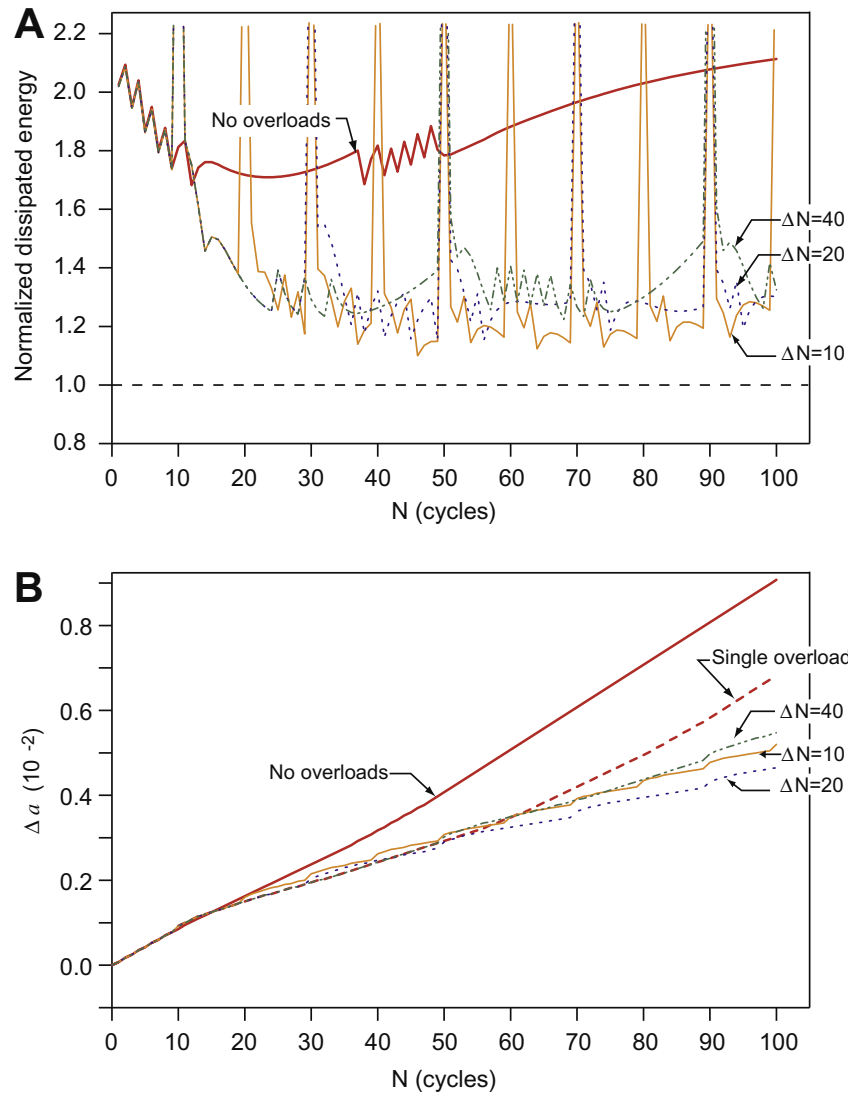

Fig. 11. For repeated overloads with $f_{0}=1.25$ : (A) normalized dissipated energy in the semi-disk domain, $W_{n}^{p}(\mathbf{D})$, after each completed load cycle, and (B) change in half crack length, $a$; as functions of the number of cycles when the overload is repeated at selected intervals $\left(W_{c r}^{p}=0.012\right)$.

tion of the crack half length, $a$, is presented in Fig. 11B. For the cases studied, we found that when the overload is applied after each 20 cycles (i.e. $\Delta N=20$ ), the crack retardation is most noticeable. The cases with $\Delta N=10$ and $\Delta N=40$ are characterized by longer crack lengths than $\Delta N=20$, Fig. 11B. When $\Delta N=10$, the crack propagation rate is influenced by that the overload is applied "too frequently" and each overload cycle results in a higher propagation increment. For $\Delta N=40$, the retardation effect induced by one overload diminishes before the following overload is applied.

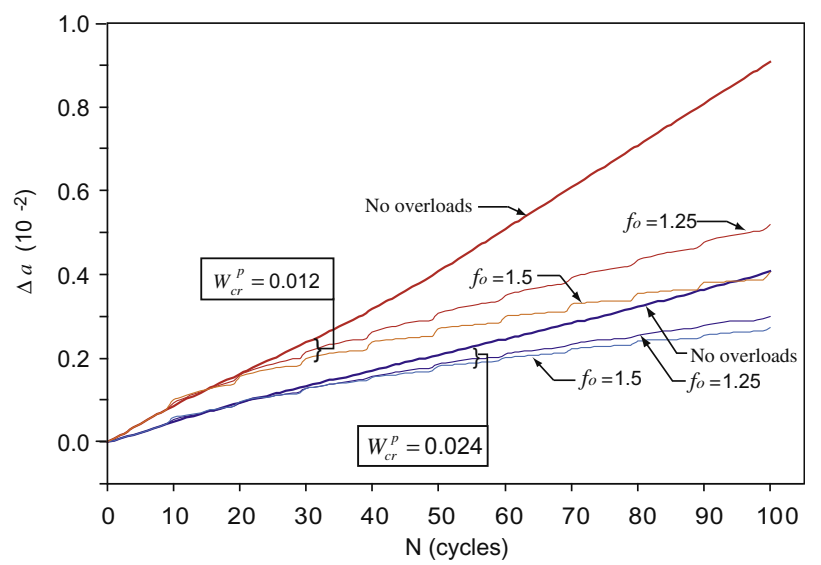

Fig. 12. Change in half crack length, $\Delta a$, as a function of the number of cycles, $N$, for repeated overloads with $f_{0}=1.25$ and $f_{0}=1.5$ shown for $W_{c r}^{p}=0.012$ and $W_{c r}^{p}=0.024$ when the overload is repeated after each 10 cycles. 
However, in all cases with repeated overloads, the final half crack length is less than for the case when only the initial overload is applied (Fig. 11B).

Finally, we investigate the effect of the critical plastically dissipated energy, considering the cases $W_{c r}^{p}=0.012$ and $W_{c r}^{p}=0.024$, for the cases of repeated overloads of $f_{0}=1.25$ and $f_{0}=1.5$. The overload is repeated after each 10 cycles and the load ratio is $R=0$ with $\bar{\sigma}_{\max }=4 / 6$. The evolution of the half crack length is shown in Fig. 12. Crack retardation is captured for all cases, but is more noticeable when $W_{c r}^{p}=0.012$. As expected, the higher overload factor $\left(f_{0}=1.5\right)$ decreases the crack propagation rate more than the case of the lower overload factor $\left(f_{0}=1.25\right)$.

\section{Concluding remarks}

A proposed condition for simulating crack propagation due to cyclic loading through a numerical scheme has been investigated qualitatively by employing the finite element method. The propagation criterion is based on a condition that relates the plastically dissipated energy to a critical value. To this end, the accumulated plastically dissipated energy is integrated over a discrete semi-disk domain in front of the crack-tip and the crack propagates when the criterion is fulfilled. Thus, the propagation rate is not specified, but results from an iterative evaluation of the propagation criterion. A higher value of the critical plastically dissipated energy results in a slower crack propagation rate.

To investigate if a propagation criterion based on the plastically dissipated energy in a domain defined in front of the crack tip is a viable condition, the effects of load ratio $\left(R=\bar{\sigma}_{\min } / \bar{\sigma}_{\max }\right)$ and of tensile overloads (with overload factor $f_{0}=\bar{\sigma}_{\text {overload }} / \bar{\sigma}_{\text {max }}$ ) on the fatigue crack growth rate were studied. Based on experimental observations, it is well established that a negative load ratio may increase the crack propagation rate. Contrarily, tensile overloads tend to decrease the crack propagation rate. The numerical results presented here suggest that the proposed scheme qualitatively captures these rate changes very well. Thus, the proposed propagation criterion appears to be a viable approach for numerically simulating crack growth due to cyclic loading where the propagation rate automatically results from simulations.

In the current form, the approach presented in this work can be used to establish relative crack propagation tendencies for different load cases. For absolute values ("real" values), calibration with physical experiments are needed. The shape and size of the integrated domain and the critically dissipated energy are material parameters in the proposed scheme. These parameters can in the simulations be made dependent (in addition to the material selected with associated properties) on any measurable quantity in the FE-model, such as (but not limited to) crack length, cycle number and time. The values used in this presentation were selected to show that the proposed concept is viable. For example, the value of the critically dissipated energy was selected solely with the purpose of achieving a reasonable propagation rate with respect to the numerical simulations and will not give a correct crack growth rate for a real system. A quantitative development and calibrating the proposed criterion requires significant experimental work, where the size and shape of the integration domain along with appropriate values for the critically dissipated energy are established. Moreover, further numerical investigations are necessary for calibrating the mesh size along the predicted path of crack propagation. All these are the topics for future work.

\section{Acknowledgment}

The authors would like to acknowledge funding from NSF DMR0710210 and the University of Delaware.

\section{References}

[1] Ellyin F. Fatigue damage, crack growth and life prediction. New York: Springer; 2001.

[2] Newman Jr JC. The merging of fatigue and fracture mechanics concepts: a historical perspective. NASA Langley Research Center; 1997.

[3] Ritchie RO. Mechanisms of fatigue-crack propagation in ductile and brittle solids. Int J Fract 1999;100(1):55-83.

[4] Schijve J. Fatigue of structures and materials. Springer; 2003.

[5] Stephens RI, Fatemi A, Stephens RR, Fuchs HO. Metal fatigue in engineering. 2nd ed. John Wiley \& Sons; 2001.

[6] Suresh S. Fatigue of materials. 2nd ed. Cambridge University Press; 1998.

[7] Krajcinovic D. Damage mechanics. Amsterdam: Elsevier Science B.V.; 1996.

[8] Lemaitre J, Desmorat R. Engineering damage mechanics: Ductile, creep, fatigue and brittle failures. Berlin: Springer-Verlag; 2005.

[9] Paris PC, Gomez MP, Anderson WP. A rational analytic theory of fatigue. Trend Eng 1961;13:9-14.

[10] Paris PC, Erdogan F. A critical analysis of crack propagation laws. J Basic Eng 1963;85:528-34.

[11] Elber W. Fatigue crack closure under cyclic tension. Eng Fract Mech 1970;2(1):37-45.

[12] Elber W. Citation classic - the significance of fatigue crack closure - damage tolerance in aircraft structures. Cc/Eng Tech Appl Sci 1983;50:22.

[13] Rice JR. The mechanics of crack tip deformation and extension by fatigue. Symp. on fatigue crack growth In: ASTM-STP-415; 1967.

[14] Bodner SR, Davidson DL, Lankford J. A description of fatigue crack growth in terms of plastic work. Eng Fract Mech 1983;17(2):189-91.

[15] Turner CE. A re-assesment of ductile tearing resistance (Part I) In: Firrao D editor. Fracture behavior and design of materials and structures, proc. ECF 8; 1990. p. 933-49.

[16] Turner, C.E., A re-assesment of ductile tearing resistance (Part II). In: Fracture behavior of materials and structures, proc. ECF 8; 1990. p. 951-68.

[17] Klingbeil NW. A total dissipated energy theory of fatigue crack growth in ductile solids. Int J Fatigue 2003;25:117-28.

[18] Cojocaru D, Karlsson AM. An object-oriented approach for modeling and simulation of crack growth in cyclically loaded structures. Adv Eng Software 2008;39(12):995-1009.

[19] Cognard JY, Ladeveze P. Talbot P. A large time increment approach for thermomechanical problems. Adv Eng Software 1999;30:583-93.

[20] Kiewel H, Aktaa J, Munz D. Application of an extrapolation method in thermocyclic failure analysis. Comput Methods Appl Mech Eng 2000;182:55-71.

[21] Fish J, Yu Q. Computational mechanics of fatigue and life predictions for composite materials and structures. Comput Methods Appl Mech Eng 2002;191:4827-49.

[22] Oskay C, Fish J. Fatigue life prediction using 2-scale temporal asymptotic homogenization. Int J Numer Methods Eng 2004;61:329-59.

[23] Cojocaru D, Karlsson AM. A simple numerical method of cycle jumps for cyclically loaded structures. Int J Fatigue 2006;28(12):1677-89.

[24] Daily JS, Klingbeil NW. Plastic dissipation in fatigue crack growth under mixed-mode loading. Int J Fatigue 2004;26:727-38.

[25] Daily JS, Klingbeil NW. Plastic dissipation in mixed-mode fatigue crack growth along plastically mismatched interfaces. Int J Fatigue 2006;28(12): 1725-38.

[26] McClung RC, Sehitoglu H. On the finite-element analysis of fatigue crack closur. 1. Basic modeling issues. Eng Fract Mech 1989;33(2):237-52.

[27] McClung RC, Sehitoglu H. On the finite-element analysis of fatigue crack closure. 2. Numerical results. Eng Fract Mech 1989;33(2): 253-72.

[28] Solanki K, Daniewicz SR, Newman JC. Finite element modeling of plasticityinduced crack closure with emphasis on geometry and mesh refinement effects. Eng Fract Mech 2003;70(12):1475-89.

[29] Zapatero J, Moreno B, Gonzalez-Herrera A. Fatigue crack closure determination by means of finite element analysis. Eng Fract Mech 2008;75(1): 41-57.

[30] ABAQUS 6.6 scripting reference manual 2006 providence. RI: ABAQUS, Inc.

[31] Cojocaru D. On numerical modeling of cyclically loaded structures. Ph.D. Dissertation. University of Delaware; 2008.

[32] Dunne F, Petrinic N. Introduction to computational plasticity. Oxford University Press; 2005.

[33] Simo JC, Hughes TJR. Computational inelasticity. Springer; 1998.

[34] Feddersen CE. Discussion to plane strain crack toughness testing. ASTM STP 1966;410:77.

[35] Solanki K, Daniewicz SR, Newman JC. Finite element analysis of plasticityinduced fatigue crack closure: an overview. Eng Fract Mech 2004;71(2):149-71.

[36] Singh KD, Parry MR, Sinclair I. Some issues on finite element modeling of plasticity induced crack closure due to constant amplitude loading. Int J Fatigue 2008;30(10-11):1898-920.

[37] Antunes FV, Rodrigues DM. Numerical simulation of plasticity induced crack closure: Identification and discussion of parameters. Eng Fract Mech 2008;75(10):3101-20.

[38] ABAQUS 6.6 user's manual. 2006, Providence. (RI): ABAQUS, Inc.

[39] Stephens RI, McBurney GW, Oliphant LJ. Fatigue crack growth with negative $R$ ratio following tensile overloads. Int J Fract 1974;10(4):587-9. 
[40] Wheeler OE. Spectrum loading and crack growth. J Basic Eng 1972;94(1):181-6.

[41] Corbly DM, Packman PF. On the influence of single and multiple peak overloads on fatigue crack propagation in 7075-T6511 aluminum. Eng Fract Mech 1973;5(2):479-96.

[42] Vardar O. Effect of single overload in FCP engineering. Fract Mech $1988 ; 30(3): 329-35$.
[43] Shercliff HR, Fleck NA. Effect of specimen geometry on fatigue crack-growth in plane-strain. 2. Overload response. Fatigue Fract Eng Mater Struct 1990;13(3):297-310.

[44] Verma BB, Pandey RK. The effects of loading variables on overload induced fatigue crack growth retardation parameters. J Mater Sci 1999:34:4867-71.

[45] Borrego LP, Ferreira JM, da Cruz JMP, Costa JM. Evaluation of overload effects on fatigue crack growth and closure. Eng Fract Mech 2003;70(11):1379-97. 